\title{
¿RASGOS PÚNICOS EN LOS SANTUARIOS IBÉRICOS? RELIGIÓN E IDENTIDAD EN LA CONTESTANIA CENTRAL
}

\begin{abstract}
Desde antes de la llegada de los ejércitos bárquidas, en el sureste peninsular se constata un fuerte influjo cultural púnico, que entre otras cosas se materializa en el campo de las manifestaciones religiosas. Este influjo ha servido a algunos autores para argumentar la presencia e intervención de gentes púnicas en la zona desde el s. IV a.C. Sin embargo, a través de los ejemplos de los templos de la Illeta dels Banyets (Campello, Alicante) y el santuario de la Serreta de Alcoi (Alcoy, Alicante), interpreto estas aparentes anomalías como materializaciones de discursos identitarios que aprovechan el medio cultural hibrido propio de un middle ground colonial para obtener unos determinados fines políticos y económicos.
\end{abstract}

Palabras clave: identidad híbrida, cartagineses, Contestania, II Edad del Hierro.

\section{Punic features in Iberian shrines? Religion and identity in Central Contestania}

From before the arrival of Carthaginian armies to the Southeast of the Iberian Peninsula, there has been a strong cultural Punic influence, materialized, among others, in the field of the religious manifestations. Some authors have focused on that influence to argue the presence and intervention of Punic people in the region since the 4th century BC. Nevertheless, I will discuss the examples of the temples of Illeta dels Banyets (Campello, Alicante) and the shrine of Serreta de Alcoi (Alcoy, Alicante). I interpret these supposed anomalies as the materialization of identity discourses constructed to take advantage of the hybrid cultural milieu typical of a colonial middle ground in order to obtain some political and economical goals.

Key words: hybrid identity, Carthaginians, Contestania, Second Iron Age.

La presencia de cartagineses en las costas del sureste peninsular con anterioridad a la conquista bárquida es uno de los aspectos más problemáticos de la arqueología ibérica. Y es que, aunque durante mucho tiempo esta presencia se consideró un hecho incontestable (Nordström 1961; Blázquez y García-Gelabert 1991: 28-32), desde los años setenta la implantación de la Nueva Arqueología y su énfasis autoctonista han llevado a negar todo contacto más allá del mero intercambio comercial (Llobregat 1981: 284286), lo que repercutió en un abandono general de su estudio que solamente se está subsanando en las dos últimas décadas ( $c f$. por ejemplo Oliver 1995; Sala 2001-2002; 2010; Bendala 2005; Ferrer y Pliego 2010).
Una de las esferas más frecuentemente argüidas para afirmar o refutar estos contactos es, sin duda, la de la religión. Aunque no es mucho lo que realmente conocemos de la religiosidad púnica (Ferrer 2004: 107), y menos aún de la ibérica, sí que nos encontramos en el sureste y levante peninsulares con toda una serie de fenómenos difíciles de explicar únicamente desde el punto de vista de la cultura ibérica, entendida como entidad autóctona y estanca. Me refiero, por ejemplo, a la temprana y masiva aparición de pebeteros con forma de cabeza femenina ( $c f$. Marín y Horn 2007), al culto betílico que parece ponerse de manifiesto en determinados recintos sacros edetanos (cf. Seco 2010), o a elementos iconográficos tales como 


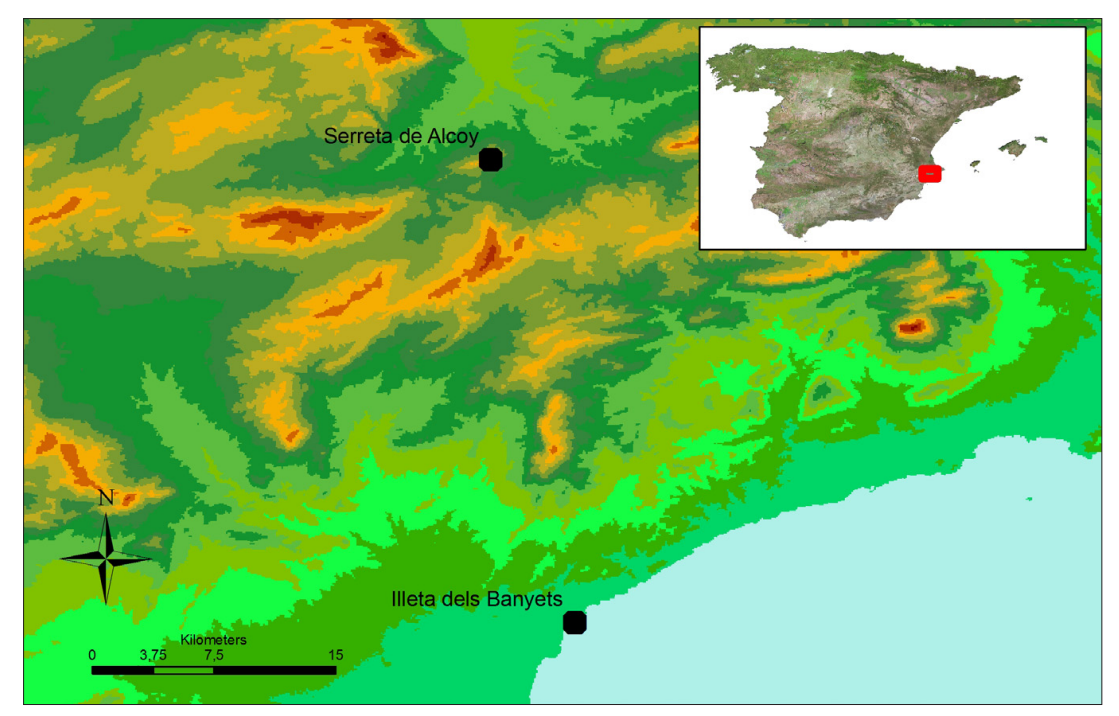

Fig. 1. Situación de los yacimientos analizados. Elaboración propia.

la esfinge del Parque Infantil de Tráfico de Elche (Chapa y Belén 2011), las divinidades aladas de la cerámica ilicitana ( $c f$. Olmos y Tortosa 2010) o el monumento turriforme grabado en el pilar-estela de Monforte del Cid (Almagro y Ramos 1986). Elementos todos ellos que en un momento $\mathrm{u}$ otro han servido como supuesto indicio de la presencia de poblaciones cartaginesas en la Península prebárquida, o para defender el impulso aculturador cartaginés sobre la religión ibérica, pero que también otros autores han intentado explicar desde la lógica interna de la religión ibérica.

La aparente dificultad parte del antiguo paradigma, ya con milenios de antigüedad y aún no del todo superado (Ruby 2006: 28-36), que daba por sentada una identidad entre cultura material y etnia. Tradicionalmente, se venía aceptando que las culturas eran entidades discretas, inmutables e impermeables, y que el cambio cultural se producía mediante un proceso unilineal, progresivo e inevitable de aculturación, adoptando gradualmente la población más atrasada los rasgos culturales de la más adelantada en el momento en que ambas entraban en contacto. Sin embargo, en las últimas décadas los estudios postcoloniales evidencian que la realidad histórica es más compleja. La cultura puede concebirse, según algunos autores, como una construcción histórica, producto y agente histórico al mismo tiempo, definible únicamente a partir y en función de las otras culturas con las que se relaciona en cada momento dado (Dietler 2009: 23-29; Gruen 2011). De esta manera, resulta ya hoy imposible pensar en diversas sociedades que mantengan culturas impermeables entre sí, ninguna sociedad puede considerarse pura en un momento dado, y todo contacto cultural provocará diversos tipos de intercambios bidireccionales de resultados heterogéneos y en muchas ocasiones inesperados que solo podrán estudiarse desde el punto de vista de lo local, dada la importancia de la agencia de los diversos grupos en contacto (Van Dommelen 1998: 33; 2004: 138; Hodos 2006: 14-15; VivesFerrándiz 2006: 168).

En todo contacto colonial (pues, en definitiva, de eso estamos hablando), no se produce el encuentro entre dos pueblos con culturas impermeables entre sí, sino que son diversos grupos sociales de distintas procedencias, cada uno con sus propios habitus, los que se encuentran, interrelacionan y conviven. Fruto de este encuentro, se genera un middle ground, un espacio de negociación cultural e identitaria en el que los distintos grupos tratarán de encontrar un mejor acomodo según sus aspiraciones e intereses, creándose, reforzándose, transformándose o eliminándose diversas identidades culturales y políticas (White 1991; Malkin 2002: 152-156; Dietler 2010: 86). Las realidades resultantes serán híbridas, esto es, radicalmente diferentes de las estructuras originales y de su adición, pero consecuentes con la nueva coyuntura histórica generada por el proceso colonial, por la agencia de los diversos grupos intervinientes, y por los intercambios desiguales generados (Van Dommelen 1997: 309).

Hablamos de contacto colonial, por cierto, refiriéndonos al sentido que para colonialismo emplean P. van Dommelen (1997: 306) y M. Dietler (2010: 18) en tanto que proyecto y práctica de control (económico, político, 
o del tipo que sea) ejercido entre sociedades caracterizadas por unas relaciones asimétricas de poder, y a los procesos de transformación social y cultural resultantes de estas prácticas. Desde este punto de vista, no es necesaria la fundación de colonias para que podamos hablar de relaciones coloniales de explotación, o de una coyuntura típicamente colonial.

Dado que se trata de una de las esferas más significativas del ámbito ideológico, en tanto que habitual sustentadora de la jerarquía social y en tanto que parte integrante del capital simbólico (Bourdieu 2009: 63-64; Edwards 2005: 113), la religión no puede evitar verse afectada por el intercambio colonial. En pocas ocasiones, sin embargo, el estudio arqueológico de lo religioso ha venido acompañado de una reflexión teórica consecuente (Insoll 1999: 1), a resultas de lo cual se ha dado por supuesto que la expansión colonial de un pueblo supondría la exportación de su religión, que sería automáticamente aceptada e integrada por las gentes colonizadas (Lane 1999: 153). Sin embargo, la ideología (y, en concreto, la religión) se transforma al mismo tiempo que la estructura de poder a la que legitima (Alvar 1993), hibridándose de una u otra forma según las necesidades de los grupos en contacto (Bhabha 1994; Aranegui y Vives-Ferrándiz 2006; Cañete y Vives-Ferrándiz 2011; Van Dommelen y Rowlands 2012; Silliman 2013). En consecuencia, nos encontraremos con una amplia casuística, a resultas de la cual no podremos colegir la etnia o la procedencia de un pueblo únicamente a partir de sus manifestaciones religiosas, pero sí profundizar en el imaginario y la estructura de poder, que se naturalizan mediante el empleo de ciertas ideas religiosas expresadas mediante un determinado conjunto de ritos y signos.

Íntimamente asociado con el fenómeno religioso y los cambios que éste experimenta en relación con el contacto colonial, habremos de prestar especial atención igualmente a la esfera de las identidades colectivas. Y es que estas corresponden asimismo con otro aspecto de la ideología, y por tanto deben entenderse como una construcción histórica que se construye y reconstruye continuamente según las circunstancias de cada momento y la agencia de los diversos grupos implicados, que hasta cierto punto pueden manipularlas consciente o inconscientemente, ya que las diversas identidades colectivas se producen y reproducen en el contexto de la interacción social (Jenkins 1997: 57-70). Desde este punto de vista instrumentalista de la etnicidad, en el que nos encuadramos, ésta puede manifestarse mediante diversos caracteres comunes (lengua, religión, territorio, antepasados comunes, etc.) (Eriksen 1993: 34-35; Hall 2002: 9-10), pero en cualquier caso será consecuente con un proyecto político determinado y con el habitus que de aquel emane (Jones 1997: 84-100). La etnicidad será empleada para legitimar (o atacar) una determinada estructura social, y por consiguiente sus manifestaciones se exacerbarán aún más cuando dicha estructura social y política se sienta amenazada, esto es, en las etapas de fuertes transformaciones (Eriksen 1993: 76; Jones 1997: 69-75; Jenkins 1997: 10; Cardete 2011: 121-122).

Una de las consecuencias que podemos extraer de lo anterior es que, al no ser la etnicidad algo inmanente a un grupo humano dado, al no ser algo natural que le viene dado, la identificación unívoca entre cultura y grupo étnico queda superada. Hablar de un grupo étnico aislado, impermeable a las influencias culturales de las sociedades que le son vecinas, es problemático (Eriksen 1993: 9-10), y aunque bien es cierto que los criterios que expresan etnicidad se suelen construir por oposición a las sociedades vecinas (Hall 2002: 9), esto no es realmente necesario. Las identidades colectivas rara vez han pretendido poseer un carácter puro y sin adulterar (Gruen 2011, especialmente pp. 223-227), y en ocasiones, si por diferentes motivos le ha interesado al grupo social dominante, una sociedad ha buscado aproximarse a otra, vincularse míticamente con ella, o incluso identificarse con ella si ello fuera menester. Independientemente de que se tratara de sociedades genéticamente distintas, o de que los historiadores contemporáneos así nos empeñemos en aseverarlo.

En definitiva, creemos que es posible estudiar ciertos rasgos punicizantes -en el sentido en el que empleó este término J. P. Morel (1986: 27)- perceptibles en la religiosidad ibérica del sureste, no como supuestos indicios por sí mismos de la presencia púnica en la región antes de la llegada de los ejércitos bárquidas, sino como caracteres culturales híbridos forjados en el encuentro colonial y que nos ofrecen una vía de aproximación hacia las estructuras de poder ibéricas y el imaginario que las generaba y sustentaba. Para ello, tomaremos como casos de estudio dos espacios del sureste que consideramos especialmente reveladores en este sentido, pues en ocasiones se los ha concebido como lugares de culto púnicos o punicizados, verdaderos vestigios, en opinión de E. Llobregat (1994) de la tradición religiosa púnica en Contestania: los templos de La Illeta dels Banyets (Campello, Alicante) y el santuario de La Serreta de Alcoi (Alicante). Dos yacimientos que hemos escogido solamente por ser los dos 
citados por Llobregat en su célebre trabajo, pero que no son sino dos de los enclaves más conocidos de toda una costa, la del sureste peninsular, en la que los ejemplos de "influencias" púnicas e hibridación cultural son constantes (cf. Sala 2010; Prados 2013).

\section{LOS TEMPLOS DE LA ILLETA DELS BANYETS (CAMPELLO, ALICANTE)}

El asentamiento ibérico de La Illeta dels Banyets (fig. 2) se extiende sobre una meseta estrecha y alargada de la costa septentrional de Campello, abarcando unas 0,4 ha conservadas. Muestra un urbanismo ortogonal, aparentemente planificado ex novo, aunque la desaparición de los límites de la meseta por erosión y la voladura de buena parte del istmo únicamente nos permiten conocer una parte del mismo (Olcina y García 1997; Olcina 2005: 149; Olcina et al.2009: 49-50). El estudio de su registro cerámico griego e indígena ha permitido fecharlo entre mediados del s. V y mediados del III a.C. (Sala 1998: 41; Garcia i Martín 2003: 31; Olcina 2005: 156-157). Por otra parte, su localización, la

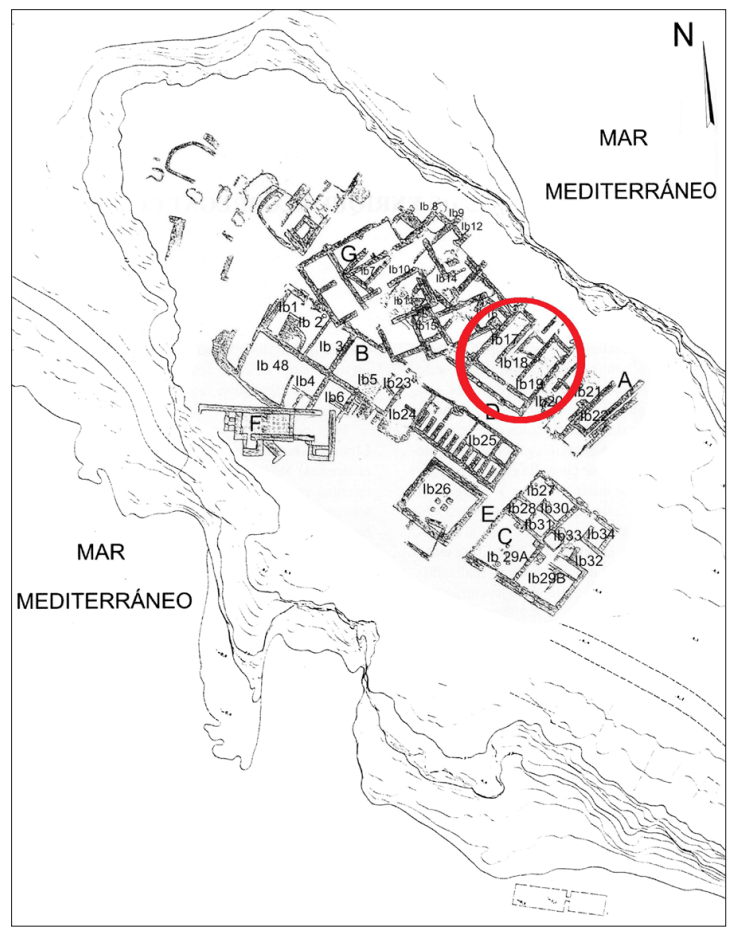

Fig. 2. Planimetría de la Illeta dels Banyets, con el "Templo A" remarcado. Elaboración propia a partir de: Olcina et al. 2010: fig. 44. abundancia de importaciones documentadas (Garcia i Martín 2003), la distribución de las ánforas producidas en el lugar (López Seguí 1997; Álvarez 1998), la presencia de un gran almacén (Álvarez 1997) y la supuesta ausencia de muralla (que recientemente viene siendo contestada: Olcina 2005: 149; Olcina et al. 2009: 213-215) ha llevado desde hace ya años a concebir este poblado como un núcleo comercial que se ocuparía de redistribuir a escala regional las importaciones mediterráneas (Llobregat 1997; Sala 2001-2002: 296).

Aparte de este marcado carácter comercial, encontramos en el poblado toda una serie de rasgos que lo convierten en extraordinario dentro de la arqueología ibérica. Uno de los más conocidos, sin duda, es la presencia de sus dos templos. El primero de ellos, conocido generalmente como "templo A" (Llobregat 1985: 103-105; 1988: 137-139; Olcina et al. 2009: 220-224) (fig. 3), es un edificio de unos $100 \mathrm{~m}^{2}$, alargado, con una pronaos, una distribución central tripartita, y dos departamentos que se abren al fondo de la estructura. Su puerta principal, flanqueada por dos columnas, se abría a la calle principal del poblado, y su parte trasera se alzaba sobre el acantilado. Frente a él, al otro lado de la calle, se ha atestiguado un almacén, que se viene considerado relacionado funcionalmente con este edificio. En el interior del "templo A" solo se han hallado algunos materiales revueltos, entre los que destacan algunos pebeteros con forma de cabeza femenina y un posible fragmento de escultura antropomorfa.

Por todo ello, generalmente se ha asignado a esta estructura una función de lugar de culto, siguiendo la interpretación que propuso su excavador (Llobregat 1985: 104). Bien es cierto que otros autores vienen considerando este edificio como una regia o estructura palacial con funciones domésticas, políticas y sacras (Almagro y Domínguez 1989: 366; Jiménez Ávila 2009), o bien como un lugar de intercambio sacralizado (Prados 2004; 2011), dependiendo de los distintos modelos interpretativos del mundo ibérico de los que parten. Sin entrar en profundidad en esta discusión, que excedería los límites del presente texto, baste al menos con subrayar la singularidad del edificio dentro del asentamiento, y su funcionalidad (importante, si es que no principal) sacra, a tenor del registro material mueble documentado.

Nos encontramos, en definitiva, con un tipo de edificación poco habitual en el mundo ibérico, para la que se han buscado reminiscencias en el mundo etrusco, púnico y próximo oriental (Llobregat 1988: 138-139; Almagro y 
Domínguez 1989: 366; Gusi 1997: 181; Olcina 2005: 150-151; Prados 2010: 60-63), por lo que se ha considerado, en ocasiones, una clara evidencia de la aculturación religiosa y política ibérica.

A pocos metros del templo A y en el centro del poblado se ubica el llamado templo B (Llobregat 1985: 106108; 1988: 140-142; Olcina 2005: 152-153; Olcina et al. 2009: 187-193), una edificación cuadrangular, de unos $50 \mathrm{~m}^{2}$, compuesta de un muro perimetral en el que se abre un solo vano y posiblemente sin techar. Atestigua dos fases constructivas diferentes, en la primera de las cuales se erigieron en su interior dos plataformas de piedra y adobe, una de las cuales pudo sostener, en opinión de su excavador, un betilo, en tanto que en la fase más reciente se erigieron dos columnas exentas, un quemaperfumes y un pebetero con forma de busto femenino.

Este tipo de arquitectura, igualmente poco frecuente en el mundo ibérico, llevó desde un primer momento a considerar este edificio como un temenos sacro al aire libre similar a los habituales en el mundo próximo-oriental y púnico (Llobregat 1985: 108; Moneo 1995: 248-249; Moratalla y Verdú 2007: 360), interpretación que se veía reforzada por los materiales registrados en su interior.

Así pues, en un mundo como el ibérico en el que los templos urbanos exentos apenas han sido atestiguados antes del s. III a.C. (cf. García Cardiel 2013), nos encontramos en Campello con dos estructuras templares de aire punicizante que parecen dominar la estructura urbana del lugar. A ello han de sumarse las particularidades del registro cerámico, en el que como ya hemos dicho las importaciones mediterráneas de todo tipo son especialmente abundantes, pero en el que además destaca la abundancia de cerámicas púnicas y ebusitanas (Sala 2001-2002: 297), la frecuencia de formas escasamente atestiguadas en el mundo ibérico como los platos de pescado, o la profusión de grafitos grecoibéricos y púnicos (Llobregat 1989). También se ha querido ver una raigambre cultural centromediterránea en la arquitectura del almacén (Bendala 2005: 42) y de los viveros de pescado construidos en torno a La Illeta (Llobregat 1986: 66), así como en la utilización del mortero hidráulico de cal en los seis lagares documentados (Sala 2010: 943). Todo ello ha llevado a que diversos investigadores propongan la existencia de un grupo de comerciantes púnicos residiendo en el lugar (Sala 2001-2002: 207; 2004: 84), a que éste sea concebido como un establecimiento comercial sometido al poder indígena pero vinculado con Massalia (Abad 2009: 29), o incluso a describirlo como un puerto de comercio administrado por los púnicos (Aranegui 2012: 212-214).

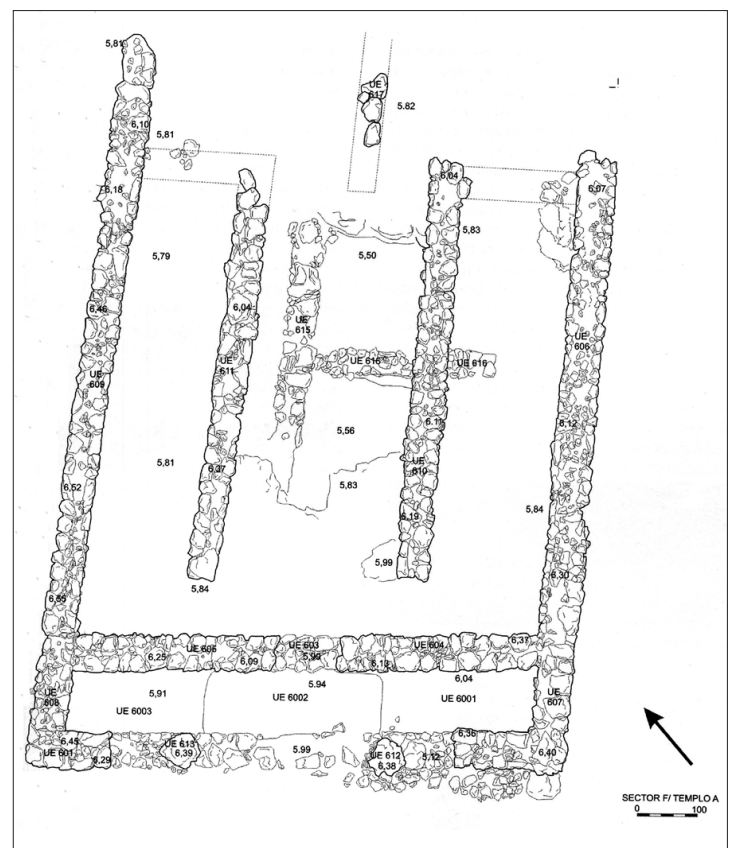

Fig. 3. Planimetría del "Templo A" de la Illeta dels Banyets. Fuente: Olcina et al. 2010: fig. 255.

Desde nuestro punto de vista, sin embargo, estas suposiciones parten de la identificación, tradicionalmente asumida como decíamos en la introducción a estas páginas, entre cultura material y etnia, identificación que consideramos por sí misma problemática.

Desde luego, la presencia de los templos en La Illeta no puede pasársenos por alto. Su importancia en la articulación del poblado (Abrams 1989; Gracia 1998: 104-105; Gruel et al. 2008: 35) debía ser manifiesta, tanto por sus grandes proporciones (y, por consiguiente, por la mano de obra que su construcción y manutención entrañaba) como por su localización, en el centro del poblado y, en uno de los casos, irguiéndose sobre el pequeño acantilado que conformaba el límite septentrional de la península, y por tanto bien visible para los barcos que recorrieran la costa alicantina siguiendo el régimen general de mareas en este sector. Además, se trata de estructuras arquitectónicas poco habituales en el mundo ibérico pero con claros paralelos mediterráneos, e igualmente se le ha asignado un carácter exógeno a algunos de los artefactos de culto documentados en su interior, como los pebeteros con forma de cabeza femenina, el quemaperfumes, la columna exenta o la imagen de culto. 
Ahora bien, un examen detallado del registro pone de manifiesto que ninguno de estos elementos constituye un hápax estricto en la arqueología ibérica. Edificios asimilables a los documentados en Campello los encontramos en la Alcudia de Elche (Alicante) (Ramos 1995) y Tres Hermanas (Aspe, Alicante) (Moratalla y García Gandía 1998-1999); pebeteros con forma de cabeza femenina fueron empleados en todo el ámbito ibérico, y con una mayor profusión en el sureste, sobre todo a partir del s. III a.C. (Moratalla y Verdú 2007); e incluso las columnas exentas podrían haber sido habituales en los santuarios del sureste ibérico, como parece poderse colegir de la arqueología y la iconografía de los mismos (García Cardiel 2013). Por lo tanto, no creemos posible afirmar con seguridad que en Campello se practicaba un tipo de culto extraño a la religiosidad ibérica, y mucho menos, por lo tanto, que éste era llevado a cabo por gentes de otra etnia.

En cambio, proponemos que nos encontramos en $\mathrm{La}$ Illeta dels Banyets ante los resultados de lo que Ch. Gosden (2008: 57-100) denominó un fenómeno de colonialismo en un medio cultural compartido. En este puerto de comercio, en el que la convivencia entre gentes de diversa procedencia y cultura debió ser continuada, no tardaron en surgir estructuras culturales híbridas, radicalmente distintas a las de partida. Las prácticas de consumo, tan estrechamente ligadas a la esfera de lo identitario (Dietler 2009: 31-32; Notario 2012), nos lo atestiguan, gracias por ejemplo a la abundancia de platos de pescado documentados en La Illeta, raros en otros asentamientos ibéricos. Es posible que este elevado grado de hibridación estuviera en realidad enmascarando la explotación colonial a la que los comerciantes llegados del Mediterráneo Central y Oriental estaban sometiendo a las gentes locales (Van Dommelen 1997: 309), pero en todo caso posiblemente esta explotación no sería percibida como tal por la mayoría de la población, y no desde luego por las elites locales, que se verían beneficiadas por estos contactos. De hecho, en una sociedad de jefaturas como la ibérica, el prestigio que el gobernante carismático adquiere gracias a sus contactos con gentes provenientes de espacios lejanos, contactos que suelen traducirse en la adquisición de bienes exóticos y de valiosa información, se constituye en una herramienta ideológica fundamental para legitimar su preeminencia social (Helms 1988). Por todo ello, no resulta extraño que una comunidad local comience a construir y expresar su identidad utilizando un lenguaje ambiguo, caracterizado por una fuerte componente exógena, fácilmente comprensible para las poblaciones mediterráneas con las que estas gentes locales estarían en continuo contacto, siempre y cuando dichos elementos exógenos puedan ser integrados en las propias estructuras locales (Van Dommelen 2002).

Así pues, independientemente del origen último, genético, de cada uno de los habitantes de La Illeta (posiblemente muy mezclado, como resulta esperable de un puerto como éste), seguramente esta comunidad desarrolló una identidad y una cultura híbridas que la diferenciaban de las poblaciones de su entorno. En este contexto puede explicarse, quizás, que estas gentes hicieran mayor hincapié en aquellos aspectos de la religiosidad ibérica más cercanos a las costumbres religiosas de los comerciantes que continuamente llegarían al puerto, y que las elites locales invirtieran grandes cantidades de recursos en la construcción de dos templos que, siendo poco frecuentes para la arquitectura ibérica tradicional, dieran cabida a este tipo de culto compartido, que sin duda facilitaría las transacciones comerciales (Domínguez 2001).

\section{EL SANTUARIO DE LA SERRETA DE ALCOI (ALICANTE)}

El poblado de La Serreta de Alcoi se sitúa sobre una elevación aislada localizada en pleno centro del AlcoiàComtat, dominando el valle del Serpis desde el que posiblemente sea el punto de mayor visibilidad de toda la cubeta intramontana (Llobregat et al. 1992: 41). Habitado desde época orientalizante (Martí y Mata 1992: 104), el asentamiento se estructura en sucesivas terrazas a lo largo de la pendiente, recorridas por calles conectadas entre sí por rampas y flanqueadas por hileras de departamentos adosados de dos plantas (Llobregat et al. 1992: 67-68). Hubo de constituir desde muy pronto un importante núcleo redistribuidor del comercio, como sugiere el importante volumen de importaciones y de inscripciones documentadas en el poblado (Garcia i Martín y Grau 1997: 121-122; Grau y Segura 1994-1995), aunque en el poblado se advierten igualmente vestigios de una importante actividad agrícola, metalúrgica y alfarera. La prosperidad resultante de estas actividades, de hecho, se trasladará a la riqueza amortizada en los ajuares funerarios de la necrópolis (Cortell et al. 1992), entre los cuales destaca la abundancia de armas (Reig 2000), propia de una sociedad cuya aristocracia dominante escogía en muchos casos representarse a sí misma como guerreros en el momento de sus rituales funerarios. 


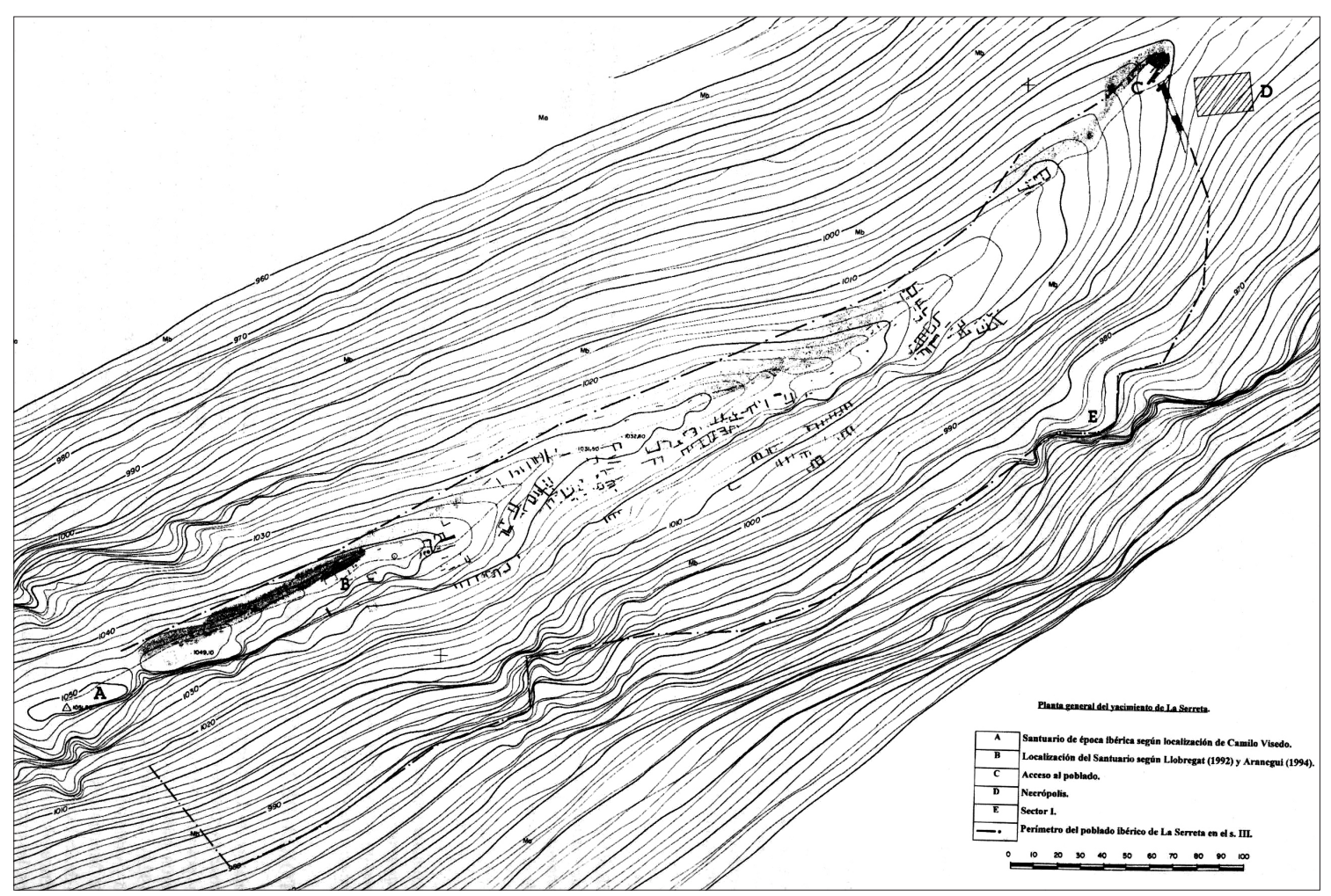

Fig. 4. Planimetría de La Serreta. Fuente: Olcina et al. 1998: 36.

Dicha prosperidad económica llevó, incluso, a que en un momento difícil de precisar entre los ss. IV y III a.C., coincidiendo con etapas de destrucción o abandono de los otros asentamientos de primer orden del Alcoià, La Serreta se erigiera en núcleo hegemónico de toda la meseta intramontana, tal y como han propuesto diversos autores (Olcina et al. 1998: 42-4; Olcina 2005: 166-167).

En la segunda mitad del s. III a.C., en todo caso, el asentamiento experimenta una notable transformación. Todo su perímetro queda rodeado de una potente muralla, parte de la cual, junto con la puerta de acceso, se construye sobre la vieja necrópolis, que de este modo queda cancelada, cesando su uso funerario (Llobregat et al. 1995: 13562). El asentamiento además crece sobremanera gracias a la construcción de varios barrios de nueva planta (Grau 1996; Olcina et al. 2000), alcanzando en conjunto las 5,5 ha de extensión, esto es, convirtiéndose en el asentamiento más grande de la región (Olcina et al. 1998: 37) (fig. 4).

Coincidiendo en el tiempo con esta transformación urbana, en la parte más alta del cerro que ocupa el poblado comienzan a depositarse centenares de exvotos de terracota, de una tipología bastante peculiar. Nos encontramos posiblemente ante un santuario suburbano, aledaño a las murallas de La Serreta e íntimamente relacionado con ésta, pues para acceder al mismo era necesario atravesar el poblado (Grau 2000: 197-198), pero cuya influencia trascendería su entorno más inmediato, pues no en vano era visible desde buena parte del valle (Grau 2010: 114117). No sabemos cuándo comenzó a emplearse como santuario esta área, pero la primera actividad cultual detectada en ella se refiere precisamente a la deposición de estos exvotos, datable en el s. III a.C. (Juan 1987-1988: 329). De hecho, la circunstancia probada de que las diversas series de terracotas se moldearan y amortizaran en un corto lapso de tiempo (Horn 2011: 164), y la coincidencia a finales del s. III a.C. de otros tipos de materiales depositados en el santuario como lucernas o monedas (Lara 2005: 125-126; Garrigós y Mellado 2004: 202204), nos llevarían a proponer que fue precisamente en las últimas décadas del s. III a.C., y no durante toda la centuria, cuando se fabrican y amortizan estos exvotos de terracota. En todo caso, a finales del mencionado siglo, 


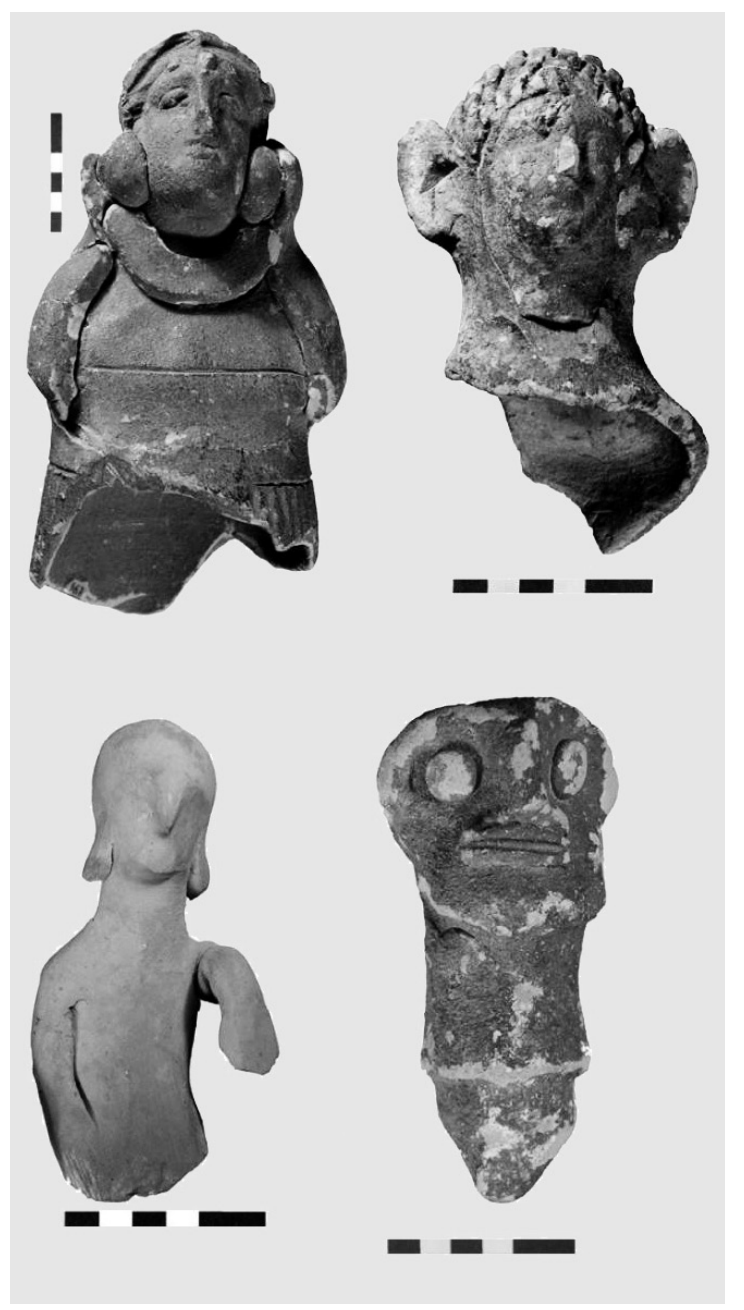

Fig. 5. Selección de terracotas del santuario de la Serreta. Fuente: Horn 2011: Anexo 1, 63, 129, 149 y 186.

quizás a consecuencia de la II Guerra Púnica, el poblado de La Serreta y sus fortificaciones fueron destruidos, y el santuario quedó aparentemente abandonado durante algún tiempo, hasta que, ya empleando una edilicia típicamente romana (Olcina 2005: 171; Grau y Garrigós 2007: 146-147), se construya una cella que atestigüe la reutilización del lugar de culto.

La deposición de pequeños exvotos representando a los propios devotos en los santuarios es una actividad ritual típicamente ibérica. No lo es tanto sin embargo el empleo de la terracota, pues se trata de un material habitualmente utilizado en los santuarios centromediterráneos pero cuya difusión por el mundo ibérico es tardía y limitada, y de hecho el de Alcoi es, de largo, el mayor conjunto de terracotas del mundo ibérico, con casi dos millares de fragmentos (Horn 2011: 151-152). Tampoco resultan habituales los tipos representados, entre los cuales la presencia de guerreros armados, varones con manto o mujeres desnudas es anecdótica, mientras que abundan tipos nada frecuentes en otros santuarios ibéricos como los exvotos-cabeza masculinos o la representación de grupos junto a la divinidad (Gusi 1997: 191-192; Aranegui y Prados 1998: 140-142) (fig. 5). Tipos para los que, de hecho, en ocasiones se ha propuesto una filiación púnica (Juan 1987-1988: 316-318), y que encuentran sus mejores paralelos en santuarios centromediterráneos, o bien en conjuntos de exvotos peninsulares tardíos para los que se ha señalado una cierta influencia púnica, como algunos de los edetanos (Bonet et al. 1990: 185199), Castellar (Ruiz Rodríguez et al. 2010: 75), La Algaida (Corzo 2007) o la Cueva del Valle (Celestino y Cazorla 2010).

Pese a estos exvotos, en todo caso, no creemos justificado defender la afluencia a La Serreta de devotos púnicos, ni la existencia en el santuario de un tipo de culto ajeno a la religiosidad ibérica. Tampoco nos encontramos, en nuestra opinión, ante otro caso como el de La Illeta dels Banyets que hemos descrito páginas atrás, en el que percibíamos un universo cultural híbrido fruto de la convivencia continuada de gentes muy diversas. La Serreta de Alcoi de finales del s. III a.C. es, dejando a un lado por un momento sus grandes dimensiones y la hegemonía que posiblemente ejerciera sobre toda su comarca, un típico asentamiento ibérico en pendiente, sin que apenas nada en su registro material nos lleve a pensar en que sus habitantes mantuvieran unos modos de vida radicalmente diferentes a los de otras comunidades ibéricas de la comarca. La abundancia de importaciones no es significativa en este sentido, pues en su gran mayoría se trata de vasos para beber y de cuencos, con formas habituales en otros asentamientos ibéricos de la región.

Únicamente se ha argüido en alguna ocasión que la fortificación del poblado, y concretamente el torreón, presentaba paralelos mediterráneos, y que de hecho estaba diseñado para contener ataques con maquinaria de asedio (Gracia 2000: 150), algo que supondría la asunción de que en el poblado había arquitectos familiarizados con la poliorcética avanzada mediterránea, y que de hecho la posibilidad de un asedio en el que se utilizara maquinaria de guerra era un hecho. No obstante, un análisis más detenido de las fortificaciones ha llevado a la 
mayoría de los autores a desechar esta idea, argumentando que se trata de estructuras ibéricas prototípicas, adaptadas a las curvas de nivel y fabricadas con el mismo tipo de paramentos y de técnicas constructivas que el resto de los edificios del poblado, sin nada que ver con el tipo de fortificaciones técnicamente más avanzadas del Mediterráneo central (Moret 2001: 142; Olcina 2005: 169).

Tampoco la escritura nos ofrece una pista en este sentido, pues pese a la abundancia de epígrafes documentados en el asentamiento, todos ellos contienen escritura ibérica levantina o greco-ibérica, pero no se dan grafitis púnicos como de los que hablábamos en Campello.

Así pues, no creemos que pueda argumentarse que en La Serreta de Alcoi existía a finales del s. III a.C. un elevado grado de hibridismo cultural que justificara la deposición en el santuario suburbano de centenares de exvotos de aspecto punizante. Ahora bien, para encontrar la clave de este fenómeno debemos detenernos, creemos, en el contexto histórico del poblado. En las últimas décadas de la tercera centuria el poder fáctico cartaginés se extendió por todo el S, SE y E peninsulares, controlando algunos enclaves y estableciendo su hegemonía sobre el resto del territorio mediante pactos y tratados. Resultado de lo cual fue, entre otras cosas, la fortificación según una concepción poliorcética avanzada (en este caso sí) del Tossal de Manises (Alicante), que funcionaría como punto fuerte avanzado cartaginés para asegurar el control de la región (Olcina et al.2010). Y resultado de ello fue también, posiblemente, la construcción apresurada de la fortificación en torno a La Serreta, pero también su inusitado crecimiento, el flujo de productos intercambiados que algunos autores creen detectar entre Serreta y Tossal de Manises (Olcina et al. 1998: 41-42), y la llegada a La Serreta de varias lucernas (Lara 2005: 125-126) y cuatro monedas (tres hispanocartaginesas y una romana: Garrigós y Mellado 2004: 202-204), todo lo cual nos está hablando de la inmersión de la antigua capital de los valles alcoyanos en el clima de inestabilidad política y militar reinante, pero también de las fluidas relaciones que la elite dirigente de La Serreta parece que se esforzó en mantener con las autoridades púnicas, sólidamente apostadas en Carthago Noua y cuyo puesto de avanzada en Tossal de Manises controlaba la salida al mar de la comarca del Alcoià a través de los puertos de Benifallim y Tudons.

Es este contexto político, desde nuestro punto de vista, el que cristaliza en el santuario de La Serreta, definido ya hace algunos años por I. Grau (2000: 198) como la manifestación ideológica de los vínculos políticos del poblado. En el clima de inestabilidad creciente (política, pero sin duda también cultural e identitaria), seguramente se tornó necesario legitimar de alguna manera los fuertes lazos establecidos entre la capital alcoyana y el poder cartaginés. Con todo ello no pretendemos defender, es de señalar, que la presencia púnica en la región constituya el detonante o factor último que motivara el desarrollo político de la Serreta; pero sí que la inestabilidad política resultante de dicha presencia favoreció el desarrollo del programa político centralizador encabezado por ésta ya desde un tiempo antes y debido a un complejo conjunto de factores (Grau 2007), y que la cooperación con el poder cartaginés fue posiblemente una de las estrategias puestas en práctica durante estas décadas por los gobernantes alcoyanos para fortalecer su poder, cooperación que era necesario justificar ideológicamente ante propios y extraños. Y para ello, quizás, se optó por revestir un tipo de ritual habitual en el mundo ibérico (la deposición de exvotos en un área sacra) de un lenguaje iconográfico punicizante. Sin reparar en que nunca antes en La Serreta se habían depositado exvotos, en que antes de que se establecieran estas relaciones que era preciso legitimar quizás el santuario no existía, o al menos sus visitas no entrañaban este gesto ritual. Para la elite gobernante de La Serreta era necesario reinventar la tradición (Hobsbawn 1983; Cuozzo 2003: 18-19) para legitimar su estrategia política (como ya propusiera recientemente I. Grau 2007: 137), y para ello nada mejor que establecer un ritual en un santuario, escenario especialmente apropiado para la manipulación ideológica que entraña la usurpación del pasado colectivo y su remplazo por una memoria más adecuada (Cardete 2005: 149); un ritual que en su forma sería típicamente ibérico, y por tanto podría ser comprendido y aceptado por la población de los valles alcoyanos, pero que se ejecutaría siguiendo un lenguaje punicizante.

Ello no quiere decir, por supuesto, que las elites gobernantes de La Serreta hubieran abrazado la cultura o la religiosidad púnicas, ni mucho menos que se hubieran punicizado. Siempre resulta difícil establecer qué elementos materiales son los que nos están marcando una identificación étnica (Fernández Götz 2009: 195), pero la ausencia de un verdadero cambio en los modos de vida del poblado creemos que en este sentido es revelador. Lo cual no debe extrañarnos, habida cuenta de que un individuo o un grupo social puede poner en práctica simultáneas dinámicas de pertenencia identitaria (Polignac 2006: 14). 


\section{CONCLUSIONES}

Como recapitulación a estas páginas, quisiéramos subrayar la idea de que los encuentros coloniales provocan una fluida negociación identitaria que engendra una infinita gama de respuestas locales. Por ello, no es posible establecer modelos mecanicistas que presupongan la aculturación sistemática de los pueblos colonizados, y su asunción progresiva de la religión y, finalmente, de la identidad de los colonizadores. Los contactos son siempre bidireccionales, dialécticos, y pese a las posiciones desiguales de las que suelen partir, no podemos olvidar nunca la agencia de los distintos grupos en liza, que condicionará siempre los resultados del encuentro.

Tampoco podemos aceptar de forma acrítica la identificación entre cultura y etnia. Aseveración ésta que de forma teórica viene siendo aceptada desde hace ya décadas por buena parte de la historiografía, pero que en lo que se refiere a la religión provoca aún problemas, al aceptarse en muchas ocasiones de forma apriorística que la documentación de un culto en un momento dado supone la presencia en el lugar de gentes provenientes del lugar de surgimiento del culto.

Los cultos, como el resto de los elementos que forman la cultura, no son entidades naturales, propias de un pueblo o de una comunidad, sino que son construcciones históricas, y por tanto abiertas a la transformación y la hibridación si la coyuntura histórica -si las necesidades de la elite que los controla- así lo requiere.

En este sentido, hemos argumentado que los templos de La Illeta dels Banyets de Campello no pueden ser tomados como evidencia de la punicidad del enclave, aunque desde luego tampoco se trata de edificios típicamente ibéricos. Más bien albergarían un tipo de culto híbrido, consecuente con las diversas gentes que posiblemente habitarían el lugar, y con el resto de la cultura material del enclave.

Por su parte, el santuario de La Serreta de Alcoi parece albergar un tipo de ritual ibérico en el fondo pero revestido de una iconografía punicizante, un ritual establecido precisamente para legitimar ideológicamente unas relaciones políticas establecidas entre la capital alcoyana y el poder hegemónico púnico en un contexto especialmente inestable.

Jorge García CARdiel Depto. de Historia Antigua Facultad de Geografía e Historia Universidad Complutense de Madrid jgarciacardiel@ucm.es

\section{AGRADECIMIENTOS}

Agradezco a S. Montero Herrero y T. Chapa Brunet, su siempre amable apoyo, respaldo, dedicación y consejo. Este trabajo se llevó a cabo en el marco del proyecto de investigación HAR2011-26096.

\section{BIBLIOGRAFÍA}

ABAD, L. (2009): Contestania, griegos e iberos, Huellas griegas en la Contestania ibérica (M. Olcina, J. J. Ramón, eds.), Alicante, 20-29.

ABRAMS, E. M. (1989): Architecture and energy. An evolutionary perspective, Archaeological method and theory 1 (M. B. Schiffer, ed.), Tucson, 47-87.

ALMAGRO, M.; DOMÍNGUEZ, A. (1989): El palacio de Cancho Roano y sus paralelos arquitectónicos y funcionales, Zephyrus 41-42, 339-382.

ALMAGRO, M.; RAMOS, R. (1986): El monumento ibérico de Monforte del Cid, Lucentum 5, 45-63.

ALVAR, J. (1993): Problemas metodológicos sobre el préstamo religioso, Formas de difusión de las religiones antiguas $(\mathrm{C}$. Blánquez, C. González Wagner, eds.), Madrid, 1-33.

ÁLVAREZ, N. (1997): El almacén del templo A: aproximación a espacios construidos especializados y su significación económica, La Illeta dels Banyets (El Campello, Alicante). Estudios de la Edad del Bronce y Época Ibérica (M. Olcina, ed.), Alicante, 133-174.

ÁLVAREZ, N. (1998): Producción de ánforas contestanas: el almacén de El Campello (Alicante), Cypsela 12, 213-226.

ARANEGUI, C. (2012): Los iberos ayer y hoy. Arqueologías y culturas, Madrid.

ARANEGUI, C.; PRADOS, L. (1998): Santuarios. El encuentro con la divinidad, Los iberos, príncipes de Occidente (C. Aranegui, ed.), Barcelona, 135-145.

ARANEGUI, C.; VIVES-FERRÁNDIZ, J. (2006): Encuentros coloniales, respuestas plurales: los ibéricos antiguos de la fachada mediterránea central, De les comunitats locals als estats arcaics: la formació de les societats complexes a la costa del Mediterrani occidental (M. C. Belarte, J. Sanmartí, eds.), Arqueomediterrània 9, 89-107.

BENDALA, M. (2005): La Contestania ibérica y el mundo púnico, La Contestania Ibérica, treinta años después (L. Abad, F. Sala, I. Grau, eds.), Alicante, 37-51.

BHABHA, H. J. (1994): The location of culture, Londres.

BLÁZQUEZ, J. M.; GARCÍA-GELABERT, M. P. (1991): Los bárquidas en la Península Ibérica, II Congresso Internazionale di Studi Fenici e Punici, Roma, 27-50.

BONET, H.; MATA, C.; GUÉRIN, P. (1990): Cabezas votivas y lugares de culto edetanos, Verdolay 2, 185-199.

BOURDIEU, P. (2009): Génesis y estructura del campo religioso, La eficacia simbólica. Religión y política (P. Bourdieu, ed.), Buenos Aires, 43-89. 
CAÑETE, C.; VIVES-FERRÁNDIZ, J. (2011): 'Almost the same'. Dynamic domination and hybrid contexts in Iron Age Lixus, Larache, Morocco, World Archaeology 43, 124-143. DOI: http://dx.doi.org/ 10.1080/00438243.2011.544909.

CARDETE, M. C. (2005): Paisajes mentales y religiosos. La frontera suroeste arcadia en épocas arcaica y clásica, Oxford.

CARDETE, M. C. (2011): El valor de la propaganda en la construcción del enemigo: Atenas y las guerras médicas, Grecia ante los Imperios. V Reunión de historiadores del mundo griego (J. M. Cortés, E. Muñiz, R. Gordillo, coords.), Sevi1la, 119-130.

CELESTINO, S.; CAZORLA, R. (2010): Un paisaje sagrado en la comarca de la Serena (Extremadura), Debate en torno a la religiosidad protohistórica (T. Tortosa, S. Celestino, eds.), Anejos de AEA 55, 83-99.

CHAPA, T.; BELÉN, M. (2011): Viaje a la eternidad. El grupo escultórico del Parque Infantil de Tráfico (Elche, Alicante), SPAL 20, 151-174.

DOI: http://dx.doi.org/10.12795/spal.2011.i20.10

CORTELL, E.; JUAN MOLTÓ, J.; LLOBREGAT, E. A.; REIG SEGUÍ, C.; SALA SELLÉS, F.; SEGURA MARTÍ, J. M. (1992): La necrópolis ibérica de La Serreta: resumen de la campaña de 1987, Estudios de arqueología ibérica y romana. Homenaje a Enrique Pla Ballester, Serie de Trabajo Varios del SIP 89, Valencia, 83-116.

CORZO, R. (2007): La coroplastia del santuario de La Algaida (Sanlúcar de Barrameda, Cádiz), Imagen y culto en la Iberia prerromana: los pebeteros en forma de cabeza femenina (M. C. Marín, F. Horn, eds.), Sevilla, 195-217.

CUOZZO, M. (2003): Reinventando la tradizione. Immaginario sociale, ideologie e rappresentazione nelle necropoli orientalizzanti di Pontecagnano, Paestum.

DIETLER, M. (2009): Colonial encounters in Iberia and the Western Mediterranean: an exploratory framework, Colonial encounters in Ancient Iberia. Phoenician, Greek and indigenous relations (M. Dietler, C. López Ruiz, eds.), Chicago, 3-48. DOI:http://dx.doi.org/10.7208/chicago/9780226148489.003.0001

DIETLER, M. (2010): Archaeologies of colonialism. Consumption, entanglement and violence in Ancient Mediterranean France, Berkely-Londres.

DOI: http://dx.doi.org/10.1525/california/9780520265516.003.0002

DOMÍNGUEZ, A. J. (2001): Los mecanismos del emporion en la práctica comercial de los foceos y otros griegos del este, Ceràmiques jònies d'època arcaica: centres de producció i comercialització al Mediterrani Occidental, Barcelona, 27-45.

EDWARDS, D. N. (2005): The archaeology of religion, The archaeology of identity. Approaches to gender, age, status, ethnicity and religion, Nueva York, 110-128.

ERIKSEN, T. H. (1993): Ethnicity and nationalism. Anthropological perspectives, Londres - Boulder.

FERNÁNDEZ GÖTZ, M. A. (2009): La etnicidad desde una perspectiva arqueológica: propuestas teórico-metodológicas, ETF II: Historia Antigua 22, 187-199.
FERRER, E. (2004): La religión púnica en Iberia: lugares de culto, El mundo púnico. Religión, antropología y cultura material (A. González Blanco, G. Matilla, A. Egea, eds.), Murcia, 107-118.

FERRER, E.; PLIEGO, R. (2010): ... Auxilium consanguineis Karthaginiensis misere: un nuevo marco interpretativo de las relaciones entre Cartago y las comunidades púnicas de Iberia, Mainake 32 (1), 525-557.

GARCÍA CARDIEL, J. (2013): De la hierogamia a la ofrenda: el contacto con la divinidad en el mundo ibérico, Mediterraneo Antico 16 (1), 277-308.

GARCIA I MARTÍN, J. M. (2003): La distribución de cerámica griega en la Contestania: el puerto comercial de La Illeta dels Banyets, Alicante.

GARCIA I MARTÍN, J. M.; GRAU, I. (1997): Les ceràmiques gregues als jaciments ibèrics de L'Alcoià i El Comtat, Recerques del Museu d'Alcoi 6, 119-130.

GARRIGÓS, I.; MELLADO, J. A. (2004): Les monedes de la Serreta: consideracions sobre la circulació monetària a les comarques meridionals del País Valencià, Recerques del Museu d'Alcoi 13, 201-226.

GOSDEN, C. (2008): Arqueología y colonialismo. El contacto cultural desde 5000 a.C. hasta el presente, Barcelona.

GRACIA, F. (1998): Arquitectura y poder en las estructuras de poblamiento ibéricas. Esfuerzo de trabajo y corveas, Los iberos, príncipes de Occidente. Estructuras de poder en la sociedad ibérica (C. Aranegui, coord.), Barcelona, 99-113.

GRACIA, F. (2000): Análisis táctico de las fortificaciones ibéricas, Gladius 20, 131-170.

DOI: http://dx.doi.org/10.3989/gladius.2000.67

GRAU, I. (1996): Estudio de las excavaciones antiguas de 1953 y 1956 en el poblado ibérico de La Serreta, Recerques del Museu d'Alcoi 5, 83-119.

GRAU, I. (2000): Territorio y lugares de culto en el área central de la Contestania ibérica, QPAC 21, 195-225.

GRAU, I. (2007): Dinámica social, paisaje y teoría de la práctica. Propuestas sobre la evolución de la sociedad ibérica en el área central del Oriente peninsular, TP 64 (2), 119-142. DOI: http://dx.doi.org/10.3989/tp.2007.v64.i2.112.

GRAU, I. (2010): Paisajes sagrados del área central de la Contestania ibérica, Debate en torno a la religiosidad protohistórica (T. Tortosa, S. Celestino, eds.), Anejos de AEA 55, Madrid, 101-122.

GRAU, I.; GARRIGÓS, I. (2007): Aproximació al poblament romà de les Valls d'Alcoi, Recerques del Museu d'Alcoi 16, 113-150.

GRAU, I.; SEGURA, J. M. (1994-1995): Las inscripciones ibéricas de La Serreta y su contexto arqueológico, Arse 28-29, 117-127.

GRUEL, K.; BERNOLLIN, V.; BROUQUIER-REDDÉ, V. (2008): Les sanctuaires, éléments structurels du territoire antique, Marqueurs des paysages et systèmes socio-économiques (R. Compatangelo-Soussignan, J. R. Bertrand, J. Chapman, P. Y. Laffont, dirs.), Rennes, 35-44.

GRUEN, E. S. (2011): Rethinking the Other in Antiquity, Princeton - Oxford. 
GUSI, F. (1997): Lugares sagrados, divinidades, cultos y rituales en el levante de Iberia, QPAC 18, 171-210.

HALL, J. M. (2002): Hellenicity. Between ethnicity and culture, Chicago - Londres.

HELMS, M. W. (1988): Ulysses' sail. An ethnographic Odyssey of power, knowledge, and geographical distance, Princeton.

HOBSBAWM, E. (1983): Introduction: inventing traditions, The invention of tradition (E. Hobsbawm, T. Ranger, eds.), Cambridge.

HODOS, T. (2006): Local responses to colonization in the Iron Age Mediterranean, Londres-Nueva York.

HORN, F. (2011): Ibères, grecs et puniques en Extrème Occident. Les terres cuites de l'espace ibérique du VIIIe au IIe siècle av. J.-C., Bibliothèque de la Casa de Velázquez, 54, Madrid.

INSOLL, T. (1999): Introduction. Research foci in archaeology and world religions, Case studies in archaeology and world religion (T. Insoll, ed.), Oxford, 1-4.

JENKINS, R. (1997): Rethinking ethnicity, Arguments and explorations, Londres.

JIMÉNEZ, J. (2009): Modelos arquitectónicos en la protohistoria del suroeste peninsular: edificios «en tridente», Santuarios, oppida y ciudades: arquitectura sacra en el origen y desarrollo urbano del Mediterráneo occidental (P. Mateos, S. Celestino, A. Pizzo, T. Tortosa, coords.), Anejos de AEA 45, 89-100.

JONES, S. (1997): The archaeology of ethnicity. Constructing identities in the past and present, Londres-Nueva York.

JUAN, J. (1987-1988): El conjunt de terracotes votives del santuari ibèric de La Serreta (Alcoi, Cocentaina, Penàguila), SAGVNTVM-PLAV 21, 295-329.

LARA, G. (2005): Lucernas de La Serreta, Recerques del Museu d'Alcoi 14, 123-142.

LLOBREGAT, E. A. (1981): Revisión del papel de los cartagineses en la Historia Antigua del País Valenciano, Primer Congreso de Historia del País Valenciano 2, Valencia, 283-290.

LLOBREGAT, E. A. (1986): Illeta dels Banyets, Arqueología en Alicante (1976-1986), Alicante, 63-67.

LLOBREGAT, E. A. (1988): Un conjunto de templos ibéricos del siglo IV a.C. hallado en las excavaciones de la isla de Campello (Alicante), Homenaje a Samuel de los Santos, Murcia, 137-143.

LLOBREGAT, E. A. (1989): Los «graffiti» en escritura grecoibérica y púnica de la Illeta dels Banyets, El Campello (Alicante), APL XIX, 149-166.

LLOBREGAT, E. A. (1994): Tradición religiosa fenicio-púnica en Contestania, El mundo púnico. Historia, sociedad y cultura (A. González Blanco, J. L. Cunchillos, M. Molina, coords.), Murcia, 169-175.

LLOBREGAT, E. A. (1997): L'Illeta dels Banyets (El Campello, Camp d'Alacant). ¿Fou un empòrion?, La Illeta dels Banyets (El Campello, Alicante). Estudios de la Edad del Bronce y Época Ibérica (M. Olcina, ed.), Alicante, 13-20 (J. Padró et al., dirs.), Homenatge a Miquel Tarradell, Barcelona, 1993, 421-428.
LLOBREGAT, E. A.; CORTELL, E.; JUAN, J.; SEGURA, J. M. (1992): El urbanismo ibérico en La Serreta, Recerques del Museu d'Alcoi 1, 37-70.

LLOBREGAT, E. A.; CORTELL, E.; JUAN, J.; OLCINA, M.; SEGURA, J. M. (1995): El sistema defensiu de la porta d'entrada del poblat ibèric de La Serreta. Estudi preliminar, Recerques del Museu d'Alcoi 4, 135-162.

LÓPEZ SEGUÍ, E. (1997): El alfar ibérico, La Illeta dels Banyets (El Campello, Alicante). Estudios de la Edad del Bronce y Época Ibérica (M. Olcina ed.), Alicante, 221-250.

MALKIN, I. (2002): A colonial middle ground: Greek, Etruscan and local elites in the Bay of Naples, The archaeology of colonialism (L. Lyons, K. Papadopoulos, eds.), Los Ángeles, 151-181.

MARÍN, M. C.; HORN, F. (eds.) (2007): Imagen y culto en la Iberia prerromana: los pebeteros en forma de cabeza femenina, Sevilla.

MARTÍ, M. Á.; MATA, C. (1992): Cerámicas de tipo fenicio occidental en las comarcas de L'Alcoià y El Comtat (Alacant), SAGVNTVM-PLAV 25, 103-117.

MONEO, M. T. (1995): Santuarios urbanos en el mundo ibérico, Complutum 6, 245-255.

MORATALLA, J.; GARCÍA GANDÍA, J. R. (1998-1999): Nuevos datos sobre arquitectura de prestigio ibérica: la regia de Las Tres Hermanas (Aspe, Alicante), Lucentum 17-18, 163-182.

MORATALLA, J.; VERDÚ, E. (2007): Pebeteros con forma de cabeza femenina de la Contestania ibérica, Imagen y culto en la Iberia prerromana: los pebeteros en forma de cabeza femenina (M.C. Marín, F. Horn eds.), Sevilla, 339-366.

MOREL, J. P. (1986): La céramique à vernis noir de Carthage, sa diffusion, son influence, CEA 18, 25-68.

MORET, P. (2001): Del buen uso de las murallas ibéricas, Gladius 21, 137-144. DOI: http://dx.doi.org/10.3989/gladius.2001.81

NORDSTRÖM, S. (1961): Los cartagineses en la costa alicantina, Alicante.

NOTARIO, F. (2012): Placeres externos, disgustos internos: percepciones de la alteridad, interacciones gastronómicas y conflictos ideológicos e identitarios en la Atenas del siglo VI a.C., Ideología, identidades e interacción en el Mundo Antiguo (M. C. del Cerro, G. Mora, J. Pascual, E. Sánchez, coords.), Madrid, 357-376.

OLCINA, M. (2005): La Illeta dels Banyets, el Tossal de Manises y La Serreta, La Contestania Ibérica, treinta años después (L. Abad, F. Sala, I. Grau, eds.), Alicante, 147-177.

OLCINA, M.; GARCIA I MARTÍN, J. M. (1997): Síntesi arqueològica, La Illeta dels Banyets (El Campello, Alicante). Estudios de la Edad del Bronce y Época Ibérica (M. Olcina, ed.), Alicante, 21-46.

OLCINA, M.; GRAU, I.; MOLTÓ, S. (2000): El sector I de La Serreta: noves perspectives sobre l'ocupació de l'assentament, Recerques del Museu d'Alcoi 9, 119-144.

OLCINA, M.; GUILABERT, A.; TENDERO, E. (2010): Lectura púnica del Tossal de Manises (Alicante), Mainake 32 (1), 229-249. 
OLCINA, M.; MARTÍNEZ CARMONA, A.; SALA, F. (2009): La Illeta dels Banyets (El Campello, Alicante). Épocas ibérica y romana. I. Historia de la investigación y síntesis de las intervenciones recientes (2000-2003), Alicante.

OLCINA, M.; GRAU, I.; SALA, F.; MOLTÓ, S.; REIG, C.; SEGURA, J. M. (1998): Nuevas aportaciones a la evolución de la ciudad ibérica: el ejemplo de La Serreta, Los iberos, príncipes de Occidente. Estructuras de poder en la sociedad ibérica (C. Aranegui, coord.), Barcelona, 35-46.

OLIVER, A. (1995): La presencia púnica en los asentamientos ibéricos: una aproximación a su problemática, III Congrès International des Études Phéniciennes et Puniques 2 (M. H. Fantar, M. Ghaki, coords), Túnez, 282-296.

OLMOS, R.; TORTOSA, T. (2010): Aves, diosas y mujeres, La Dama de Baza: un viaje femenino al Más Allá (T. Chapa, I. Izquierdo, coords.), Madrid, 243-257.

POLIGNAC, F. de (2006): Espaces de communication et dynamiques d'appartenance en Grèce archaïque, L'individu et la communauté. Regards sur les identités en Grèce Ancienne (F. de Polignac, P. Schmitt-Pantel, coords.), REA 108 (1), 9-24.

PRADOS, F. (2004): ¿Almacenes o centros redistribuidores de carácter sacro? Una reflexión en torno a un modelo arquitectónico tipificado en la protohistoria mediterránea, El mundo púnico. Religión, antropología y cultura material (A. González, G. Matilla, A. Egea, eds.), Murcia, 173-180.

PRADOS, F. (2010): Una propuesta de caracterización de las llamadas regiae ibéricas. Comercio, religión y control territorial a partir de un modelo arquitectónico, Lucentum 29, 57-80.

PRADOS, F. (2013): Cartago y el mundo ibérico. Presencias y apariencias púnicas en el sureste hispano, Fragor Hannibalis. Aníbal en Hispania (M. Bendala, ed.), Madrid, 356-379.

RAMOS, R. (1995): El templo ibérico de La Alcudia. La Dama de Elche, Elche.

REIG, C. (2000): El armamento de la necrópolis ibérica de La Serreta de Alcoi (Alicante, España), Gladius 20, 75-117. DOI: http://dx.doi.org/10.3989/gladius.2000.65

RUBY, P. (2006): Peuples, fictions? Ethnicité, identité ethnique et sociétés anciennes, L'individu et la communauté. Regards sur les identités en Grèce Ancienne (F. de Polignac, P. Schmitt-Pantel, coords.), REA 108 (1), 25-60.

RUIZ RODRÍGUEZ, A.; RUEDA, C.; MOLINOS, M. (2010): Santuarios y territorios iberos en el Alto Guadalquivir, Debate en torno a la religiosidad protohistórica (T. Tortosa, S. Celestino, eds), Anejos de AEA 55, Madrid, 65-81.
SALA, F. (1998): Los problemas de caracterización del siglo III a.C. en yacimientos de la Contestania, Les fàcies ceramiques d'importació a la costa ibèrica, les Balears i les Pitiüses durant el segle III a.C. i la primera meitat del segle II a.C. (J. Ramón, J. Sanmartí, D. Asensio, J. Principal, eds.), ArqueoMediterrània 4, 29-48.

SALA, F. (2001-2002): Para una revisión de las relaciones púnicas con la costa ibérica alicantina: nuevas perspectivas sobre algunos viejos problemas, AnMurcia 16-17, 283-300.

SALA, F. (2004): La influencia del mundo fenicio y púnico en las sociedades autóctonas del Sureste peninsular, Colonialismo e interacción cultural. El impacto fenicio púnico en las sociedades autóctonas de Occidente, Ibiza, 57-102.

SALA, F. (2010): Nuevas perspectivas sobre las relaciones púnicas con la costa ibérica del Sureste peninsular, Mainake 32 (2), 933-950.

SECO, I. (2010): Piedras con alma. El betilismo en el mundo antiguo y sus manifestaciones en la Península Ibérica, Sevilla.

SILLIMAN, S. W. (2013): What, where, and when is hibridity, The archaeology of hybrid material culture (J. J. Card, ed.), Carbondale, 486-500.

VAN DOMMELEN, P. (1997): Colonial constructs: colonialism and archaeology in the Mediterranean, Culture, contact and colonialism (Ch. Gosden, ed.), World Archaeology 28 (3), 305-323.

DOI: http://dx.doi.org/10.1080/00438243.1997.9980350.

VAN DOMMELEN, P. (1998): On colonial grounds. A comparative study of colonialsm and rural settlement in first millennium B.C. west central Sardinia, Leiden.

VAN DOMMELEN, P. (2002): Ambigous matters: colonialism and local identities in Punic Sardinia, The archaeology of colonialism (L. Lyons, K. Papadopoulos, eds.), Los Ángeles, 121-147.

VAN DOMMELEN, P. (2004): Colonial interactions and hybrid practices, The archaeology of colonial encounters. Comparative perspectives (G. J. Stein, ed.), Santa Fe, 109-141.

VAN DOMMELEN, P.; ROWLANDS, M. (2012): Material concerns and colonial encounters, Materiality and social practice: transformative capacities of intercultural encounters (J. Maran, W. Stockhammer, eds.), Oxford, 20-31.

VIVES-FERRÁNDIZ, J. (2006): Negociando encuentros. Situaciones coloniales e intercambios en la costa oriental de la Península Ibérica (ss. VIII-VI a.C.), Barcelona.

WHITE, R. (1991): The Middle Ground: Indians, Empires and Republics in the Great Lakes Region, 1650-1815, Cambridge. 